\title{
ON SPACES WITH NORMS OF NEGATIVE AND POSITIVE ORDER ${ }^{1}$
}

\author{
GIDEON PEYSER
}

\begin{abstract}
The two Hilbert spaces $H_{0}$ and $H_{1}$ are defined to be a generating pair if $H_{1}$ is a dense subspace of $H_{0}$ arid if the norm of an element in $H_{1}$ is greater than or equal to the norm in $H_{0}$. It is shown that the pair generates a sequence of spaces $\left\{H_{k}\right\},-\infty<$ $k<\infty$, such that any two spaces of the sequence form again a generating fair. Such a pair is shown to generate, in turn, a subsequence of $\left\{H_{k}\right\}$. Also, representation theorems are derived for bounded linear functionals over the spaces of the sequence $\left\{H_{k}\right\}$, generalizing the Lax representation theorem and the Lax-Milgram theorem.
\end{abstract}

1. Introduction. We consider two Hilbert spaces, $H_{0}$ with norm \|\|$_{0}$ and inner product $(,)_{0}$, and $H_{1}$ with norm \|\|$_{1}$ and inner product $(,)_{1}$, having the following properties. $H_{1}$, as a vector space, is isomorphic to a subspace $\hat{H}_{1}$ of $H_{0} ; \hat{H}_{1}$ is dense in $H_{0}$; if $x_{0} \in \hat{H}_{1}$ corresponds to $x_{1} \in H_{1}$ then $\left\|x_{0}\right\|_{0} \leqq\left\|x_{1}\right\|_{1}$.

We shall identify the spaces $H_{1}$ and $\hat{H}_{1}$ but will consider their elements equipped with two sets of norms and inner products. Hence we have for $x \in H_{1}$ that

$$
\|x\|_{0} \leqq\|x\|_{1} \text {. }
$$

The spaces $H_{0}$ and $H_{1}$, with the above properties, will be called a generating pair. $H_{1}$ is the stronger and $H_{0}$ the weaker space of the pair.

In $\$ 2$ we construct from the generating pair a sequence of Hilbert spaces $\left\{H_{k}\right\},-\infty<k<\infty$, such ihat any two spaces $H_{k_{1}}$ and $H_{k_{2}}, k_{1}<k_{2}$, form a generating pair with $H_{k_{1}}$ the weaker and $H_{k_{2}}$ the stronger space. In [3] Lax considers the space $G$ of square integrable functions and the space $G^{(r)}$ of functions with square integrable derivatives up to order $r . G$ and $G^{(r)}$ form, in our notation, a generating pair. Lax constructs the space $G^{(-r)}$,

Presented to the Society, January 19, 1972 under the title On negative and positive norms; received by the editors March 28, 1971.

AMS 1970 subject classifications. Primary 46C10; Secondary 46B10.

Key words and phrases. Hilbert space, norm, inner product, weaker space, stronger space, lower space, upper space, isometric isomorphism, correspondence, bounded linear functional, representation theorem.

${ }^{1}$ This paper was supported in part by the NSF grant GP-8601. 
the space of functions with norms of negative order. These spaces play an important role in existence and regularity theorems in partial differential equations. For further details regarding these negative norms, see Schechter [6] and Yosida [7]. In [1] Landesman constructs, in a generalized way, spaces that correspond to the negatively normed spaces of Lax, and analyzes their properties.

The main results are in $\$ 3$ where we show that the sequence of $\left\{H_{k}\right\}$ is closed in the following sense. Any two spaces of the sequence generate a subsequence of $\left\{H_{k}\right\}$. We also derive representation theorems for bounded linear functionals over the spaces of the sequence $\left\{H_{k}\right\}$, which generalize the Lax representation theorem [3] and the Lax-Milgram theorem [4]; see also Landesman [2].

2. Generated spaces. For fixed $x \in H_{0}$ we consider the linear functional over $H_{1}, g(y)=(y, x)_{0}, y \in H_{1}$. Then

$$
|g(y)|=\left|(y, x)_{0}\right| \leqq\|x\|_{0}\|y\|_{0} \leqq\|x\|_{0}\|y\|_{1} .
$$

Hence $g(y)$ is a bounded linear functional over $H_{1}$. It follows from the Riesz representation theorem that there exists a unique $z \in H_{1}$ such that

$$
g(y)=(y, x)_{0}=(y, z)_{1} .
$$

We define the negative norm of $x$ by

$$
\|x\|_{-1}=\|z\|_{1} \text {. }
$$

To justify this designation of a norm we have to show that if $x_{0} \in H_{0}$ with $\left\|x_{0}\right\|_{-1}=0$ then $x_{0}=0$. Indeed, $\left\|x_{0}\right\|_{-1}=0$ implies $\left(y, x_{0}\right)_{0}=0$ for all $y \in H_{1}$. Since $H_{1}$ is dense in $H_{0}$ it follows that $\left\|x_{0}\right\|_{0}=0$ and $x_{0}=0$.

$H_{-1}$ is the space obtained by completion under the negative norm (4) of the elements of $H_{0} . H_{-1}$ is isometrically isomorphic to a closed subspace, say $\tilde{H}_{1}$, of $H_{1}$. We show that $\tilde{H}_{1}$ exhausts all of $H_{1}$.

LEMMA $1 . \tilde{H}_{1}=H_{1}$.

Proof. Let $y_{0} \in H_{1}$ be orthogonal to $\tilde{H}_{1}$. Then $\left(y_{0}, z\right)_{1}=0$ for all $z \in \tilde{H}_{1}$. Therefore $\left(y_{0}, x\right)_{0}=0$ for all $x \in H_{0}$ and in particular for $y_{0}$. Hence $\left\|y_{0}\right\|_{0}=0$. Since $H_{1}$, as a vector space, is a subspace of $H_{0}$ we conclude that also $\left\|y_{0}\right\|_{1}=0$. This implies that $\tilde{H}_{1}=H_{1}$.

The inner product for elements $w_{1}, w_{2}$ in $H_{-1}$ is defined by

$$
\left(w_{1}, w_{2}\right)_{-1}=\left(y_{1}, y_{2}\right)_{1}
$$

where $y_{1}, y_{2}$ are the elements in $H_{1}$ corresponding respectively to $w_{1}, w_{2}$, in the preceding isometric isomorphism between $H_{1}$ and $H_{-1}$. This completes the construction of $H_{-1}$ as a Hilbert space.

Definition. $\quad H_{-1}$ is defined as the lower space generated by $H_{0}$ and $H_{1}$. 
Symbolically this will be denoted by

$$
H_{-1}=H_{0} / H_{1} \text {. }
$$

It follows from (3) that if $x \in H_{0}, y \in H_{1}$ then $\left|(y, x)_{0}\right| \leqq\|y\|_{1}\|x\|_{-1}$. Hence the inner product in $H_{0}$ can be extended boundedly to $y \in H_{1}$ and $w \in H_{-1}$, such that $(y, w)_{0}$ satisfies the generalized Schwarz inequality

$$
\left|\left(y, w^{\prime}\right)_{0}\right| \leqq\|y\|_{1}\|w\|_{-1} .
$$

Furthermore, if $w_{1}, w_{2} \in H_{-1}$ correspond respectively to $y_{1}, y_{2} \in H_{1}$, then

$$
\left(w_{1}, w_{2}\right)_{-1}=\left(y_{1}, w_{2}\right)_{0}=\left(y_{2}, y_{2}\right)_{1} \text {. }
$$

From (8) follow the dual relationships for $w \in H_{-1}$ and $y \in H_{1}$,

and

$$
\|w\|_{-1}=\sup _{Y \in H_{1}}\left\{(Y, w)_{0} \mid /\|Y\|_{1}\right.
$$

$$
\|y\|_{1}=\sup _{W \in H_{-1}} \mid(y, W)_{0} ! /\|W\|_{-1} .
$$

LEMMA 2. If $x \in H_{0}$ then $\|x\|_{-1} \leqq\|x\|_{0}$.

Proof. If $z \in H_{1}$ corresponds to $x$ as an element in $H_{-1}$, then $\|x\|_{-1}^{2}=$ $(x, x)_{-1}=(z, x)_{0} \leqq\|z\|_{0}\|x\|_{0} \leqq\|z\|_{1}\|x\|_{0}=\|x\|_{-1}\|x\|_{0}$. Hence $\|x\|_{-1} \leqq\|x\|_{0}$.

Since $H_{0}$ is dense in $H_{-1}$ it follows from Lemma 2 that $H_{-1}$ and $H_{0}$ form a generating pair. They generate the lower space which we denote by $H_{-2}$, in the same way that $H_{-1}$ is generated from $H_{0}$ and $H_{1}$. Therefore $H_{-2}=$ $H_{-1} / H_{0}$. Successively we now construct the spaces $H_{-2}, H_{-3}, H_{-4}, \cdots$, such that $H_{-k}, k>0$, is the lower space generated by $H_{-k+1}$ and $H_{-k+2}$; i.e. $H_{-k}=H_{-k+1} / H_{-k+2}$.

Next we construct from $H_{0}$ and $H_{1}$ the space $H_{2}$ as follows. $H_{2}$ is the subspace of $H_{1}$ for whose elements $z$ there exist corresponding elements $x \in H_{0}$ such that, for all $y \in H_{1}$,

$$
(z, y)_{1}=(x, y)_{0} .
$$

Since (11) can be satisfied for any $x \in H_{0}$, it follows that the correspondence between $z \in H_{2}$ and $x \in H_{0}$ is a mapping from $H_{2}$ onto $H_{0}$. Furthermore $x=0$ if and only if $z=0$ and therefore this is a one-to-one mapping.

The norm and inner product in $\mathrm{H}_{2}$ are defined by

$$
\|z\|_{2}=\|x\|_{0} \text { and }\left(z_{1}, z_{2}\right)_{2}=\left(x_{1}, x_{2}\right)_{0} \text {, }
$$

where $z, z_{1}, z_{2} \in H_{2}$ correspond respectively to $x, x_{1}, x_{2} \in H_{0}$.

It follows from (12) that the correspondence between $\mathrm{H}_{0}$ and $\mathrm{H}_{2}$ is an isometric isomorphism.

$H_{2}$ is dense in $H_{1}$. This follows from the fact that if $y \in H_{1}$ is orthogonal 
in $H_{1}$ to the elements of $H_{2}$, then (11) implies that $(x, y)_{0}=0$ for all $x \in H_{0}$. Hence $\|y\|_{0}=0$ and therefore $\|y\|_{1}=0$.

LEMMA 3. If $z \in H_{2}$ then $\|z\|_{1} \leqq\|z\|_{2}$.

Proof. If $x \in H_{0}$ corresponds to $z \in H_{2}$ then it follows from (11) that $\|z\|_{1}^{2}=(z, z)_{1}=(x, z)_{0} \leqq\|x\|_{0}\|z\|_{0} \leqq\|x\|_{0}\|z\|_{1}$. Hence $\|z\|_{1} \leqq\|x\|_{0}=\|z\|_{2}$.

Definition. $H_{2}$ is defined as the upper space generated by $H_{0}$ and $H_{1}$. Symbolically this will be denoted by

$$
H_{2}=H_{1} / H_{0}
$$

Since $H_{2}$ is dense in $H_{1}$ it follows from Lemma 3 that $H_{1}$ and $H_{2}$ form a generating pair. We generate therefore successively the spaces $H_{2}, H_{3}$, $H_{4}, \cdots . H_{k+2}$ is the upper space generated by $H_{k}$ and $H_{k+1}$. Hence $H_{k+2}=$ $H_{k+1} / H_{k}, k>0$.

To complete the construction of all the generated spaces we will show that the processes of generating lower spaces and upper spaces are reciprocal to each other. We do this by showing that $H_{1}$ is the upper space generated by $H_{-1}$ and $H_{0}$, and that $H_{0}$ is the lower space generated by $H_{1}$ and $H_{2}$.

LEMMA 4. $H_{1}=H_{0} / H_{-1}$.

Proof. Denote $H_{0} / H_{-1}$ by $H_{1}^{\prime}$. Then it is to be shown that $H_{1}^{\prime}=H_{1}$. If $y \in H_{1}^{\prime}$ corresponds to $w \in H_{-1}$ in the isometric isomorphism between $H_{-1}$ and $H_{1}^{\prime}$, then $(w, x)_{-1}=(y, x)_{0}$ for all $x \in H_{0}$. Let $W, X \in H_{1}$ correspond respectively to $w$ and $x$ as elements in $H_{-1}$, in the isometric isomorphism between $H_{1}$ and $H_{-1}$. Then $(w, x)_{-1}=(W, X)_{1}=(W, x)_{0}$. Hence $y=W$ and therefore $y \in H_{1}$. Also, $y$ as an element both in $H_{1}$ and $H_{1}^{\prime}$ corresponds to the same element $w \in H_{-1}$. Conversely if $y \in H_{1}$ then for all $x \in H_{0},(y, x)_{0}=$ $(y, X)_{1}=(w, x)_{-1}$ and therefore $y \in H_{1}^{\prime}$. It follows that $H_{1}=H_{1}^{\prime}$ which concludes the proof.

LeMmA 5. $H_{0}=H_{1} / H_{2}$.

Proof. Denote $H_{1} / H_{2}$ by $H_{0}^{\prime}$. If $y \in H_{1}$, then for all $z \in H_{2},(z, y)_{1}=$ $(z, Y)_{2}$ where $Y \in H_{2}$ corresponds to $y \in H_{0}^{\prime}$ in the isometric isomorphism between $H_{2}$ and $H_{0}^{\prime}$. Also, $(z, y)_{1}=(x, y)_{0}=(z, W)_{2}$ where $x, y \in H_{0}$ correspond respectively to $z, W \in H_{2}$ in the isometric isomorphism between $H_{2}$ and $H_{0}$. Hence $Y=W$. Therefore, $y$ as an element in both $H_{0}^{\prime}$ and $H_{0}$ corresponds to the same element $Y \in H_{2}$. Since $H_{1}$ is dense in both $H_{0}^{\prime}$ and $H_{0}$, it follows that $H_{0}^{\prime}=H_{0}$.

Summing up the properties of the spaces of the sequence $\left\{H_{k}\right\}$ we have:

THEOREM 1. For any integer $k,-\infty<k<\infty$,

(a) The spaces $H_{k}$ and $H_{k+1}$ form a generating pair, with $H_{k}$ the weaker and $H_{k+1}$ the stronger space. 
(b) $H_{k-1}=H_{k} / H_{k+1}$ and $H_{k+1}=H_{k} / H_{k-1}$.

(c) The inner product in $H_{k}$ can be extended boundedly to elements $x \in H_{k-1}$ and $z \in H_{k+1}$, and $(z, x)_{k}$ satisfies the Schwarz inequality:

$$
\left|(z, x)_{k}\right| \leqq\|z\|_{k+1}\|x\|_{k-1} .
$$

(d) There exists an isometric isomorphism between $H_{k-1}$ and $H_{k+1}$, uniquely determined by the relationships

$$
\left(x_{1}, x_{2}\right)_{k-1}=\left(z_{1}, x_{2}\right)_{k}=\left(z_{1}, z_{2}\right)_{k+1}
$$

where $x_{1}, x_{2} \in H_{k-1}$ correspond to $z_{1}, z_{2} \in H_{k+1}$.

3. Main theorems. The correspondence of the elements $x \in H_{k-1}$ and $z \in H_{k+1}$ under the isometric isomorphism of Theorem 1 will be denoted by

$$
\left(x ; H_{k-1}\right) \leftrightarrow\left(z ; H_{k+1}\right) .
$$

Now, (16) generates successively an isometric isomorphism between $H_{k-m}$ and $H_{k+m}, m>0$, similarly denoted by $\left(z ; H_{k-m}\right) \leftrightarrow\left(Z ; H_{k+m}\right)$, through the mappings

$$
\left(z ; H_{k-m}\right) \leftrightarrow\left(z_{1} ; H_{k-m+2}\right) \leftrightarrow \cdots \leftrightarrow\left(z_{m-1} ; H_{k+m-2}\right) \leftrightarrow\left(Z ; H_{k+m}\right) .
$$

LemMA 6. For integer $k$ and positive integer $m$, the spaces $H_{k}$ and $H_{k+m}$ form a generating pair with $H_{k}$ the weaker and $H_{k+m}$ the stronger space.

Proof. $H_{k+\imath n}$, as a linear vector space, is a subspace of $H_{k}$. Also, $\|z\|_{k} \leqq\|z\|_{k+m}$ for $z \in H_{k+m}$. Hence we only have to show that $H_{k+m}$ is dense in $H_{k}$. Consider first $H_{k+2}$. Since $H_{k+1}$ is dense in $H_{k}$ and $H_{k+2}$ is dense in $H_{k+1}$, it follows that for $\varepsilon>0$ and any $x \in H_{k}$ there exists $y \in H_{k+1}$ with $\|y-x\|_{k}<\varepsilon$, and $z \in H_{k+2}$ with $\|z-y\|_{k+1}<\varepsilon$. Hence $\|z-x\|_{k} \leqq\|z-y\|_{k}+$ $\|y-x\|_{k} \leqq\|z-y\|_{k+1}+\|y-x\|_{k}<2 \varepsilon$. Therefore $H_{k+2}$ is dense in $H_{k}$. It follows similarly that $H_{k+m}$ is dense in $H_{k}$ which completes the proof.

Next we show that two corresponding elements in $H_{k}$ and $H_{k+2}$ are also corresponding elements in $H_{k-1}$ and $H_{k+1}$.

Lemma 7. If $\left(x ; H_{k}\right) \leftrightarrow\left(z ; H_{k+2}\right)$, then $\left(x ; H_{k-1}\right) \leftrightarrow\left(z ; H_{k+1}\right)$.

Proof. Let $Z \in H_{k+1}$ be such that $\left(x ; H_{k-1}\right) \leftrightarrow\left(Z ; H_{k+1}\right)$. We have to show that $Z=z$. For any $y \in H_{k+1}$ let $Y \in H_{k+2}$ correspond to $y$ as an element in $H_{k}$, that is $\left(y ; H_{k}\right) \leftrightarrow\left(Y ; H_{k+2}\right)$. It follows that $(x, y)_{k}=(Z, y)_{k+1}$ and also $(x, y)_{k}=(z, Y)_{k+2}=(z, y)_{k+1}$. Hence $(Z, y)_{k+1}=(z, y)_{k+1}$. Therefore $Z=z$.

From repeated application of Lemma 7 follows

LeMma 8. If $\left(x ; H_{k}\right) \leftrightarrow\left(z ; H_{k+2}\right)$, then

$$
\left(x ; H_{k-r}\right) \leftrightarrow\left(z ; H_{k-r+2}\right) \text { for } r>0 .
$$


LEMMA 9. If $x \in H_{k+r}, r>0$, and $\left(x ; H_{k}\right) \leftrightarrow\left(z ; H_{k+2}\right)$, then $z \in H_{k+r+2}$ and $\left(x ; H_{k+r}\right) \leftrightarrow\left(z ; H_{k+r+2}\right)$.

Proof. Let $Z \in H_{k+r+2}$ be such that $\left(x ; H_{k+r}\right) \leftrightarrow\left(Z ; H_{k+r+2}\right)$, then it follows from Lemma 8 that $\left(x ; H_{i}\right) \leftrightarrow\left(Z ; H_{k+2}\right)$. Hence $Z=z$ which completes the proof.

Following Lemma $6, H_{k}$ and $H_{k+m}, m>0$, form a generating pair. We have therefore the lower space $H_{k} / H_{k+m}$ and the upper space $H_{k+m} / H_{k}$. We now have the following result:

THEOREM 2. $H_{k} / H_{k+m}=H_{k-m}$.

Proof. Denote $H_{k} / H_{k+m}=H_{k-m}^{\prime}$. Then it is to be shown that $H_{k-m}^{\prime}=$ $H_{k-m}$. Let $x \in H_{k}$. Then there exists $y \in H_{k+m}$ such that

$$
(x, w)_{k}=(y, w)_{k+m} \text { for all } w \in H_{k+m} .
$$

It follows from the definition of $H_{k-m}^{\prime}$ that $\left(x ; H_{k-m}^{\prime}\right) \leftrightarrow\left(y ; H_{k+m}\right)$. We shall show that also $\left(x ; H_{k-m}\right) \leftrightarrow\left(y ; H_{k+m}\right)$. There exist

such that

$$
x_{1} \in H_{k+1}, x_{2} \in H_{k+2}, \cdots, x_{m-1} \in H_{k+m-1}, x_{m} \in H_{k+m}
$$

$$
(x, w)_{k}=\left(x_{1}, w\right)_{k+1}=\left(x_{2}, w\right)_{k+2}=\cdots=\left(x_{m-1}, w\right)_{k+m-1}=\left(x_{m}, w\right)_{k+m}
$$

for all $w \in H_{k+m}$. It follows from (18) that $x_{m}=y$. Therefore

$$
\left(y ; H_{k+m}\right) \leftrightarrow\left(x_{m-1} ; H_{k+m-2}\right) \text { and }\left(x_{m-1} ; H_{k+m-1}\right) \leftrightarrow\left(x_{m-2} ; H_{k+m-3}\right) \text {. }
$$

Hence from Lemma $7\left(x_{m-1} ; H_{k+m-2}\right) \leftrightarrow\left(x_{m-2} ; H_{k+m-4}\right)$ and therefore $\left(y ; H_{k+m}\right) \leftrightarrow\left(x_{m-2} ; H_{k+m-4}\right)$. Next, $\left(x_{m-2} ; H_{k+m-2}\right) \leftrightarrow\left(x_{m-3} ; H_{k+m-4}\right)$ and hence $\left(x_{m-2} ; H_{k+m-4}\right) \leftrightarrow\left(x_{m-3} ; H_{k+m-6}\right)$ and therefore $\left(y ; H_{k+m}\right) \leftrightarrow$ $\left(x_{m-3} ; H_{k+m-6}\right)$. Successively it follows that $\left(y ; H_{k+m}\right) \leftrightarrow\left(x ; H_{k-m}\right)$.

Hence the elements of $H_{k}$ as a subspace of $H_{k-m}^{\prime}$ are identical to these elements as a subspace of $H_{k-m}$. Since the elements of $H_{k}$ are dense in $H_{k-m}^{\prime}$ by definition, and are dense in $H_{k-m}$ by Lemma 6 , it follows that $H_{k-m}^{\prime}=H_{k-m}$.

COROLlaRY 2.1. $H_{k+m} / H_{k}=H_{k+2 m}$.

Proof. Since Theorem 2 implies that $H_{k+m} / H_{k+2 m}=H_{k}$, it follows from Lemma 4 that $H_{k+m} / H_{k}=H_{k+2 m}$.

The following corollaries are an immediate consequence of Theorem 2.

Corollary 2.2. $H_{k}$ and $H_{k+m}$ generate the sequence $\left\{H_{k+s m}\right\},-\infty<$ $s<\infty$.

COROLLARY 2.3. The inner product in $H_{k}$ can be extended boundedly to 
elements $x \in H_{k-m}$ and $z \in H_{k+m}$ such that

$$
\left(x_{1}, x_{2}\right)_{k-m}=\left(z_{1}, x_{2}\right)_{k}=\left(z_{1}, z_{2}\right)_{k+m}
$$

where $\left(x_{1} ; H_{k-m}\right) \leftrightarrow\left(z_{1} ; H_{k+m}\right)$ and $\left(x_{2} ; H_{k-m}\right) \leftrightarrow\left(z_{2} ; H_{k+m}\right)$.

We now derive two generalized representation theorems.

TheOrem 3 (LAX RePresentation TheOREM). For any integer $k$ and positive integer $m$, all bounded linear functionals $g(z)$ over $H_{k+m}$ are uniquely represented by

$$
g(z)=\left(z, x_{g}\right)_{k}, \quad x_{g} \in H_{k-m},
$$

and all bounded linear functionals $h(x)$ over $H_{k-m}$ are uniquely represented by

$$
h(x)=\operatorname{conj}\left(z_{h}, x\right)_{k}, \quad z_{h} \in H_{k+m} .
$$

Proof. By the Riesz representation theorem $g(z)=\left(z, z_{g}\right)_{k+m}$ with $z_{g} \in H_{k+m}$ uniquely determined. It follows from Corollary 2.3 that $g(z)=$ $\left(z, x_{g}\right)_{k}$ where $\left(x_{g} ; H_{k-m}\right) \leftrightarrow\left(z_{g} ; H_{k+m}\right)$, and $x_{g}$ is uniquely determined in $H_{k-m}$. The representation $h(x)=\left(z_{h}, x\right)_{k}^{-}$is similarly established.

Theorem 4 (Generalized LaX-Milgram Theorem). Let $B(z, x)$ be $a$ form defined for all elements $z \in H_{k+m}, x \in H_{k-m}$, which is linear in $z$, antilinear in $x$, and satisfies $|B(z, x)| \leqq M\left\|z_{i k+m}^{i} \mid x\right\|_{k-m}$ for some constant $M$. Suppose that for some positive constant $c$, all pairs $z_{0}, x_{0}$, such that $\left(z_{0} ; H_{k+m}\right) \leftrightarrow\left(x_{0} ; H_{k-m}\right)$, satisfy $\left|B\left(z_{0}, x_{0}\right)\right| \geqq c\left\|z_{0}\right\|_{k+m}^{2}=c\left\|x_{0}\right\|_{k-m}^{2}$. Then every bounded linear functional $G(z)$ over $H_{k+m}$ admits a unique representation $G(z)=B\left(z, x_{G}\right), x_{G}=H_{k-m}$, and every bounded linear functional $F(x)$ over $H_{k-m}$ admits a unique representation $F(x)=\operatorname{conj} B\left(z_{F}, x\right), z_{F} \in H_{k+m}$.

Proof. We adapt the method of the proof of the Lax-Milgram theorem (see for example Nirenberg [5]) to the present circumstances. For fixed $x \in H_{k-m}, B(\mathrm{z}, x)$ is a bounded linear functional over $H_{k+m}$. Hence by Theorem 3 there exists a unique $u \in H_{m-k}$ such that $B(z, x)=(z, u)_{k}$. This defines a linear mapping $u=\mathscr{A} x$ from $H_{k-m}$ into itself. We substitute $v$ for $z$, where $\left(v ; H_{k+m}\right) \leftrightarrow\left(x ; H_{k-m}\right)$. This implies that $c\|x\|_{k-m}^{2} \leqq|B(v, x)|=$ $\left|(v, u)_{k}\right| \leqq\|v\|_{k+m}\|u\|_{k-m}=\|x\|_{k-m}\|u\|_{k-m}$. Hence $\|x\|_{k-m} \leqq c^{-1}\|u\|_{k-m}$. It follows that the operator $\mathscr{A}$ has a bounded inverse. Therefore the range of $\mathscr{A}$ is closed and every element $x \in H_{k-m}$ corresponds to a unique $u$ in the range of $\mathscr{A}$. To complete the proof it remains to show that the range of $\mathscr{A}$ is all of $H_{k-m}$. Assume that $v_{0} \in H_{k+m}$ is orthogonal to the range of $\mathscr{A}$, that is $\left(v_{0}, u\right)_{k}=0$, for all $u$ in the range. Let $x_{0}=H_{k-m}$ be such that $\left(x_{0}\right.$; $\left.H_{k-m}\right) \leftrightarrow\left(v_{0} ; H_{k+m}\right)$ and let $u_{0}=\mathscr{A} x_{0}$. Then $0=\left|\left(v_{0}, u_{0}\right)_{k}\right|=\left|B\left(v_{0}, x_{0}\right)\right| \geqq$ $c\left\|v_{0}\right\|_{k+m}^{2}$. Hence $\left\|v_{0}\right\|_{k+m}=0$ and therefore the zero element is the only element in $H_{k+m}$ orthogonai to the range of $\mathscr{A}$ (in $H_{k-m}$ ). It now follows 
from Corollary 2.3 that the range of $\mathscr{A}$ is all of $H_{k-m}$. The proof of the representation of the bounded linear functionals over $H_{k-m}$ follows by a similar argument.

\section{REFERENCES}

1. E. M. Landesman, Hilbert-space methods in elliptic partial differential equations, Pacific J. Math. 21 (1967), 113-131. MR 35 \#806.

2. - A generalized Lax-Milgram theorem, Proc. Amer. Math. Soc. 19 (1968), 339-344. MR 37 \#1965.

3. P. D. Lax, On Cauchy's problem for hyperbolic equations and the differentiability of solutions of elliptic equations, Comm. Pure Appl. Math. 8 (1955), 615-633. MR 17, 1212.

4. P. D. Lax and A. N. Milgram, Parabolic equations, contributions to the theory of partial differential equations, Ann. of Math. Studies, no. 33, Princeton Univ. Press, Princeton, N.J., 1954, pp. 167-190. MR 16, 709.

5. L. Nirenberg, Remarks on strongly elliptic partial differential equations, Comm. Pure Appl. Math. 8 (1955), 649-675. MR 17, 742.

6. M. Schechter, Negative norm.s and boundary problems, Ann. of Math. (2) 72 (1960), 581-593. MR 23 \#A2636.

7. K. Yosida, Functional analysis, Die Grundlehren der math. Wissenschaften, Band 123, Academic Press, New York; Springer-Verlag, Berlin, 1965. MR 31 \#5054.

Department of Mathematics, Newark College of Engineering, Newark, NEW JERSEY 07102 